\title{
Design of a Four-Channel Surface Receiver Coil Array without Preamplifiers for the Decoupling between Elements: Validation for high-resolution Rat Knee MR Imaging
}

\author{
A.L. Perrier, J.C. Goebel, A. Pinzano, E. Roeder, P. Gillet, D. Grenier and O. Beuf
}

\begin{abstract}
In Magnetic Resonance Imaging (MRI), multichannel coil arrays are increasingly being used to improve Signal-to-Noise Ratio (SNR) in order to increase spatial and/or temporal image resolution. Recent decoupling technique allows conception of two-channel surface transceiver coil arrays; this technique based on common conductor does not require additional preamplifier for the decoupling between elements. In this case the coil array loops are directly connected to the independent transmit/receive switches and preamplifiers of the MR system. Using this common conductor decoupling technique, a topology of a four-channel coil array was developed and described in this paper. A four-channel surface receiver coil array was designed to perform the simultaneous acquisition of both rat knee joints at $7 \mathrm{~T}$. Without the use of additional preamplifiers, a good decoupling between channels was obtained and very high spatial resolution $3 \mathrm{D}$ images with a voxel size of $49 \times 49 \times 98 \mu^{3}$ were achieved in $1 \mathrm{~h} 22 \mathrm{~min}$ scan time. Acquisitions allowed the quantification of cartilage morphological parameters such as thickness and volume.
\end{abstract}

Index Terms - small animal MRI, tunable device, fourchannels NMR surface coil array.

Manuscript received September 25, 2012.

A. L. Perrier is with the Universite de Lyon, CREATIS, CNRS UMR 5220, Inserm U1044, INSA-Lyon, Université Lyon1, Villeurbanne, France (corresponding author to provide phone: +33 (0)4 724315 97; e-mail: anne-laure.perrier@univ-lyon1.fr).

J. C. Goebel is with the Université de Lyon, CREATIS, CNRS UMR 5220, Inserm U1044, INSA-Lyon, Université Lyon1, Villeurbanne, France (e-mail: jean-christophe.goebel@creatis.insa-lyon.fr).

A. Pinzano is with the PPIA, CNRS UMR 7561, Nancy Université, Vandoeuvre-lès-Nancy, France (e-mail: astrid.pinzano@medecine.uhpnancy.fr).

E. Roeder is with the PPIA, CNRS UMR 7561, Nancy Université, Vandoeuvre-lès-Nancy, France (e-mail: emilie.roeder@medecine.uhpnancy.fr).

P. Gillet is with the PPIA, CNRS UMR 7561, Nancy Universite, Vandoeuvre-lès-Nancy, France (e-mail: pierre.gillet@medecine.uhpnancy.fr).

D. Grenier is with the Universite de Lyon, CREATIS, CNRS UMR 5220, Inserm U1044, INSA-Lyon, Université Lyon1, Villeurbanne, France (email: denis.grenier@univ-lyon1.fr).

O. Beuf is with the Universite de Lyon, CREATIS, CNRS UMR 5220,

Inserm U1044, INSA-Lyon, Université Lyon1, Villeurbanne, France (email: olivier.beuf@univ-lyon1.fr).

This work is funded by ANR with grant ref. ANR-09-BLANC-0150-01 and is conducted in the framework of the LabEX PRIMES ("Physics Radiobiology Medical Imaging and Simulation)

\section{INTRODUCTION \\ THE concept of the Nuclear Magnetic Resonance (NMR) phased array also called multi-channel coil} array was introduced in 1990 [1] to increase sensitivity of MR coils while preserving a large volume of exploration. The basic idea is to use the juxtaposition or the overlapping of several single loop coils. These coils are decoupled from each other for simultaneous and independent data acquisition [2], [3]. The use of several smaller coils increase the signal sensitivity and the Signal-to-Noise Ratio (SNR) compared with a single large loop resonator covering the same sensitive area. Another major benefit of NMR phased-array coils is the possibility to use parallel imaging techniques, such as SENSE [4] and GRAPPA [5], to accelerate the imaging process.

To build multi-channel surface coil arrays, the juxtaposition or overlapping of several single loop coils [1], [6] is mainly used for clinical human examinations [7], [8]; More recent papers described multi-channel coils with up to 128 elements. These techniques are also developed for small-animal imaging but with an inferior number of channels with typically four elements [2]. While preclinical research systems are offering multiple receiver channel capabilities, coil arrays are not systematically and still not so largely used worldwide due to the number of magnetic field available for preclinical MR imaging and due to other alternatives such as cryogenic transmit-receive surface coil.

For both human and small animal applications, phased array coils designed with several single loop elements are usually connected with low input impedance and low noise preamplifiers to reduce the coupling between the different elements of the array and to preserve SNR to its maximum respectively [1]. With a matching network, the low input impedance of the preamplifier is transformed into high impedance at the loop input thus reducing the loop current. This in turn leads to a lower inter-element coupling. In the other direction, input impedance of the loop is matched to the preamplifier to obtain a minimum of noise and ensure a good SNR at this first stage of amplification [9]. Other preamplifier stages including in MR systems are necessary to increase signal amplitude before digital conversion. However, this decoupling technique using impedance mismatch does not allow the use of the coil in transmit mode. Transmit mode requires a high current which generates coupling between 
elements. As a result, the original resonance of the coil is split in two leading to an important reduction of coil sensitivity at the operating frequency.

In human whole body systems (except at $7 \mathrm{~T}$ ), surface coil arrays are mostly used in receive mode combined with a volume coil (such as the saddle coil, birdcage coil, TEM) for transmission. In the high-field regime, when the wavelength of the associated Larmor radio-frequency (RF) approaches the dimensions of the coil and volume of interest (VOI), the volume coils become less effective. Indeed at $7 \mathrm{~T}$, the proton Larmor frequency is $300 \mathrm{MHz}$; the wavelength is $1 \mathrm{~m}$ in the air and $11 \mathrm{~cm}$ in a dielectric with an 80 effective permeability. Propagation phenomena can not be neglected anymore in human body: destructive interferences can appear due to the short wavelength in front of VOI. Thus, at high magnetic field strengths, the potential benefits of surface coil arrays for combining transmit and receive (transceiver) operations are worth exploring. Unfortunately the low input impedance preamplifier technique to reduce coupling between multiple elements of surface restrain the coil array use in receive mode only. To achieve transceiver operation, recent works described strip transmission line array [10], overlap geometries [11] and capacitive decoupling networks [11]-[14].

A different solution was described in particular for twochannel coil arrays [15]. In this setup, single elements of the array are not juxtaposed or overlapped but elements are electrically jointed with a common conductor. This technique is easier to implement than the overlapping process for small sized coil arrays, thus explaining its use for small animal applications. In this arrangement, decoupling between elements is achieved by the common conductor in series with a capacitor. Loop inputs of the array are directly connected to the independent transmit/receive switches and preamplifiers integrated to the MR system: low input impedance preamplifiers for the decoupling between elements are not required allowing the use of the coil array in transceiver mode. Based on this technique, two-channel transceiver coil arrays were developed by Gareis et al. [16]. In order to resist high voltages occurring in transmit mode, mechanical variable capacitors are used for loops matching. To our knowledge, few works used this technique for human applications or for coil array with more than two loops. A recent paper described a four-channel human cardiac transceiver coil array operating at $7 \mathrm{~T}$ with two rectangular loops placed on anterior and two placed on posterior of the body [17]. Each part is composed of a two-channel coil array based on a common conductor decoupling. The large distance between the two sets of elements insures decoupling between them. The authors took advantage of the surface coils compared to volume coil in the high-field regime. This work shows that the described decoupling technique is of interest to design transceiver coil arrays and/or for high magnetic-field applications. Another study presents a four-channel surface coil array based on common conductor decoupling between elements for rat head MR imaging [18]. The elements are aligned and placed side by side. Each neighbor element is joined by pair with a common conductor. Because the two extremity channels needed further decoupling with the preamplifier technique, the array was operating in receive mode only.
In this paper we present new principle and design of a fourchannel coil array which does not use overlapping technique but is instead on a common conductor decoupling: the four elements are lined up and joined on the same device. Low input impedance preamplifiers and external capacitive decoupling network are not required for the cross decoupling between elements. The challenge of this work is to ensure decoupling between elements directly by the adjustment of capacitors constituting the loops. To our knowledge, this coil array represents the first four-channel NMR surface coil array with these characteristics. The main objective of this paper is to present a simple but realistic equivalent electrical circuit to explain and predict the S-parameters of the coil array. The equivalent electrical circuit of the four-channel surface coil array is described in section II. Validation of this equivalent electrical circuit was performed with experimental confrontation by measuring corresponding S-parameters of a coil array designed for a dedicated small animal application. A four-channel surface receiver coil array is designed to perform the simultaneous acquisition of both rat knee joints at $7 \mathrm{~T}$. Indeed, in a previous work [19], simultaneous imaging of rat knee joints was performed with a pair of two-channel receiver coil arrays. A two-channel receiver coil array was duplicated and placed on each knee. A small sheet of copper inserted at equal distance between the two arrays was used to insure decoupling between inner elements. This previous setup did not give satisfaction due to a lack of reproducibility. We present in section III of this paper the design of the fourchannel receiver coil array with a particular wave-like topology to fit with both rat knees. The S-parameter measurements for coil elements as well as high spatial resolution MR images of rat knee cartilage are presented in section IV.

\section{EQUIVALENT ElECTRICAL CIRCUIT}

In this section we first look back on the two-channel coil array equivalent electrical circuit described in [20], it will be used to explain the four-channel coil array behavior.

\section{A. The Two-Channel Coil Array}

In a previous work [19], the equivalent electrical circuit for a two-channel coil array based on a common conductor was developed. This circuit reported in Fig. 1 a) is again described as an introduction to four-channels. Two loops constituting two basic magnetic dipoles are linked by a common $L_{c}-C_{c}$ section constitute the two-channel coil array. At the operating frequency, the $L_{c}-C_{c}$ section shorts the two loops (at the black point) by deporting the ground plane.

$$
\left|j L_{c} \omega\right|=\left|\frac{1}{j C_{c} \omega}\right|
$$

with $\omega=2 \pi f_{0}$, where $f_{0}$ is the operating frequency.

When condition (1) is met, each loop is independent of each other; a zero of transmission is achieved between the two ports ensuring decoupling between elements. At the operating frequency, the equivalent electrical circuit of each loop is then described by Fig. 1 b). Equations to match each loop to $50 \Omega$ at the operating frequency $f_{0}$ were extracted from the equivalent circuit of this figure. To match the real and the 
imaginary parts of the coil input impedance, two equations linking $R, L_{b}, C_{e}$ and $C_{a}$ values are given by:

$\frac{R}{\left(1-L_{b} C_{e} \omega^{2}\right)^{2}+\left(R C_{e} \omega\right)^{2}}=50$

$\frac{L_{b} \omega-R^{2} C_{e} \omega-L_{b}{ }^{2} C_{e} \omega^{3}}{\left(1-L_{b} C_{e} \omega^{2}\right)^{2}+\left(R C_{e} \omega\right)^{2}}-\frac{1}{C_{a} \omega}=0$

The resistor $R$ represents the sum of conductor losses, magnetic losses and series resistances of lumped elements. The $L_{b}$ inductor represents the loop inductance. $L_{c}$ inductor is the sum of the common conductor and the mutual inductance between the two loops. In practice, $L_{b}$ and $L_{c}$ is governed by the copper strip dimensions: the length of copper strip depends on the coil dimension fixed by the imaging VOI on the animal. The $C_{a}$ and $C_{e}$ capacitors are adjusted to match each loop to $50 \Omega$ at the operating frequency $f_{0}$.

Quality factor $Q$ is an important parameter to characterize a coil. For a single coil element $Q=\omega . L / R$ where $L$ is the loop inductance of the coil and $R$ its resistance. Note that quality factor measured at $50 \Omega$ and called $Q_{L}$ (for loaded quality factor) is given by $Q_{L}=Q / 2$ [21], [22]. For two coils jointed with a conductor, quality factor equation is more complex. It depends not only of $L_{b}$ and $R$ but also on $L_{c}$ and on the neighbor coil. Indeed equations (1), (2) and (3) are just valid at the operating frequency. Except for this frequency, the entire circuit of Fig. 1 a) must be considering. a)

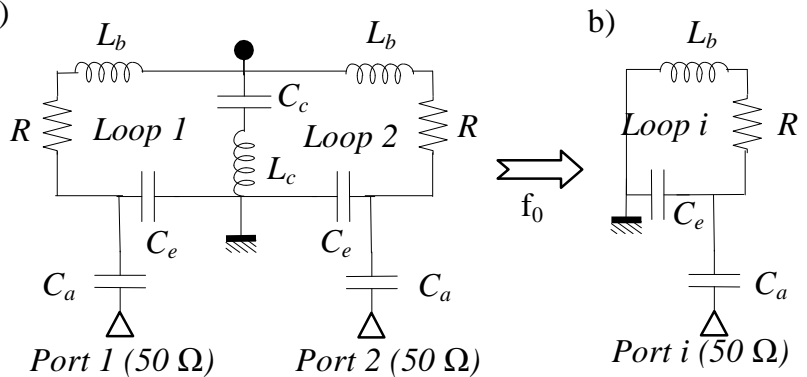

topology to conceive a four-channel coil array with a common conductor between two neighbor elements. Each inductor $L_{b}$ in Fig. 1 is divided into three new different inductors $L_{d}, L_{b}, L_{c}$ representing the upper, lower and external sides of each twochannel coil loop, respectively. Mutual inductances between two loops are implicitly included on $L_{c}$ inductors; to simplify all $L_{c}$ inductors are fixed to the same value. So mutual inductance values are not taken into account in the circuit.

The four-channel coil array topology is based on a twochannel coil array (loops 2-3); each loop is duplicated on both sides by a mirror effect giving loops 1 and 4 . At the operating frequency, decoupling between elements is achieved by the $L_{c^{-}}$ $C_{c}$ and $L_{d}-C_{d}$ sections when these sections respect condition (1). Indeed, each ground plane of Fig. 2 is deported in the black point 1 though the $L_{c}-C_{c}$ section, next it is deported from point 1 to point 2 though the $L_{d}-C_{d}$ section. Finally it is deported from point 2 to point 3 though another $L_{c}-C_{c}$ section. These perfect theoretical reports of ground plane in point 3 guarantee a prefect decoupling between channels at the operating frequency. In this case, each loop of Fig. 2 has the same equivalent electrical circuit than each loop of the two-channel coil array described in Fig. 1 b). Equations (2) and (3) remain also valid to match each four-channel coil array element to $50 \Omega$ at the operating frequency.

\section{DESIGN}

A four-channel surface coil array was designed for simultaneous imaging of both rat knee joints on a Bruker 7T Biospec MR scanner (Bruker, Ettlingen, Germany). The coil array was implemented in hybrid technology on a flexible substrate (Rogers ${ }^{\mathrm{TM}}$ RT/Duroid® ${ }^{\circledR}$ 5880, thickness: $508 \mu \mathrm{m}$ ) to fit the animal's morphology. Loops were matched to $50 \Omega$ at the proton Larmor frequency in a $7 \mathrm{~T}$ static magnetic field; i.e. $f_{0}=300 \mathrm{MHz}$ (Fig. 3).

Loops inductors were realized with $35 \mu \mathrm{m}$ thickness and $4 \mathrm{~mm}$ width section of copper tape. The lengths were fixed by the imaging volume of interest corresponding to the rat knees coverage $(22 \mathrm{~mm} \times 80 \mathrm{~mm})$. Each element of the array consists in a rectangular loop with $14 \times 15 \mathrm{~mm}^{2}$ internal and $22 \times 23 \mathrm{~mm}^{2}$ external dimensions. The flexible substrate was glued on two parallel Plexiglas ${ }^{\mathrm{TM}}$ cylinders with $21 \mathrm{~mm}$ outer diameter and $19 \mathrm{~mm}$ inner diameter leading to wave-like shaped coil to fit the animal morphology. To decouple the receiver array from the transmitter coil, each loop integrates
Fig. 1 Principle of a two-channel NMR coil array. a) The two loops are jointed by a common $L_{c}$ - $C_{c}$ section. b) At the operating frequency $f_{0}$, the $L_{c}-C_{c}$ section is shorted. $C_{e}$ and $C_{a}$ match each loop to $50 \Omega$.

\section{B. $\quad$ The Four-Channel Coil Array}

Based on the two-channel scheme, we propose, in Fig. 2, a

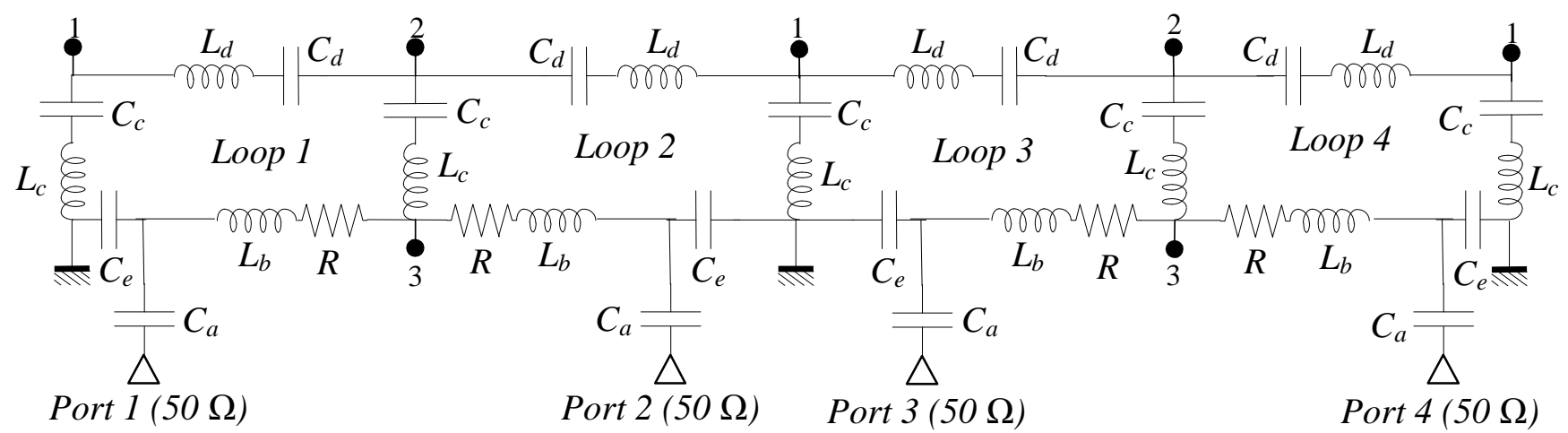

Fig. 2 Equivalent electrical circuit of a four-channel NMR coil array. At the operating frequency $f_{0}$, the $L_{c}-C_{c}$ and $L_{d}$ - $C_{d}$ sections are shorted; each loop is equivalent to Fig $1 \mathrm{~b}$ ) 
an active decoupling circuit made with one DH80055 PIN diode (Temex Ceramics, Pessac, France). This PIN diode was placed in series with the $L_{b}$ inductor to detune each loop during the transmitting phase; A $-32 \mathrm{~V}$ and $3.8 \mathrm{~V}$ bias voltage values were applied to shift off and on resonance, respectively, each coil array element. Four $50 \Omega$ BNC cables with $50 \mathrm{~cm}$ length were soldered at the four loops inputs to connect each coil of the array with the acquisition data cabinet integrating preamplifiers and transmit/receive switches of the MR system. In a previous work, it has been demonstrated that two of the three $L_{b}, C_{a}$ and $C_{e}$ elements must be adjustable to be able to tune the real and imaginary parts of each loop input impedance [20].

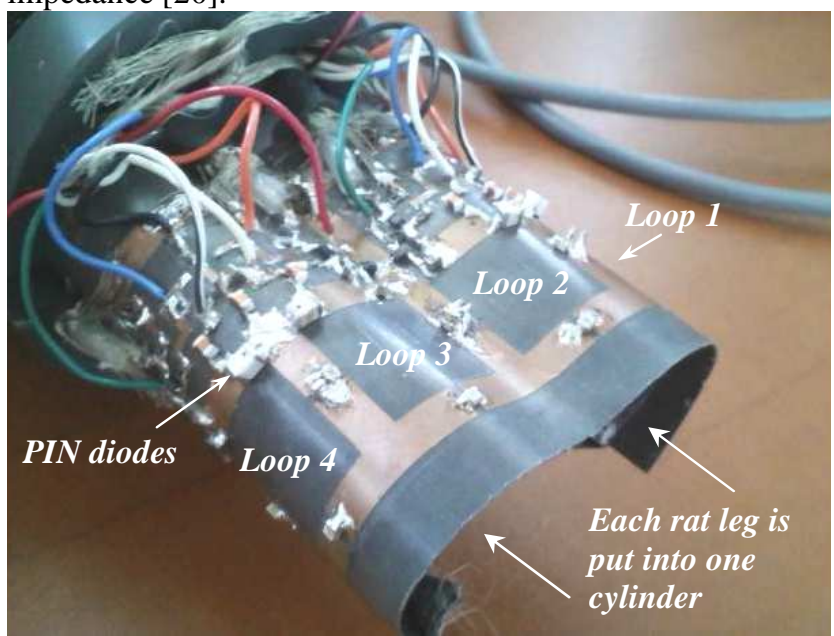

Fig. 3 Photograph of the four-channel NMR surface coil array.

The $C_{a}$ and $C_{e}$ were chosen to be the adjustable elements for the four-channel coil array. Matching of each loop to $50 \Omega$ at $f_{0}=300 \mathrm{MHz}$ was realized using varicap diodes BB149 (Philips
Semiconductors, Eindhoven, Netherlands). The capacitance values of these diodes are range from $2 \mathrm{pF}$ to $22 \mathrm{pF}$ for a bias voltages between $-30 \mathrm{~V}$ and $0 \mathrm{~V}$. Fixed capacitors are non magnetic case A series 100 and 700 ATC capacitors (American Technical Ceramics, New York, USA). The values are adjusted to minimize all $\left|S_{i j}\right|_{\mathrm{dB}}$ parameters. The adjustment procedure using fixed capacitors is fastidious. Indeed, the modification of one capacitance value alters the sixteen Sparameters. Although the determination of capacitance values through calculations (equations 2 and 3 ) and circuit simulation (section IV A) is a trivial matter when all other components (inductors and resistors) are known, experimentally it is a very challenging task. This difficulty rises from the high sensitivity of capacitance values versus inductors and resistors which are not perfectly known experimentally. Up to now we do not have a systematic method to determine and adjust capacitance values; obtaining a coil array with a good decoupling between elements is a tedious and time-consuming task.

The four-channel coil array layout is shown in Fig. 4 with the following lumped components: PIN diodes for diode and varicap diodes for variable capacitor. Only the RF circuit is presented with all copper tape dimensions and experimental values of lumped components. The direct-current (DC) circuits are represented by the "DC bias" blocks. All highfixed capacitor values (220 and $510 \mathrm{pF}$ ) are used to stop DC voltages.

We note that capacitors placed on common conductors and corresponding to $C_{c}$ in the Fig. 2 do not have the same values on the layout. Indeed $C_{c}$ capacitors are adjusted to compensate $L_{c}$ witch correspond to the sum of conductor inductance and mutual inductance between loops. Mutual inductance values no taken into account explained this difference of $C_{c}$ values.

A bias box was built to drive the DC bias voltages for each

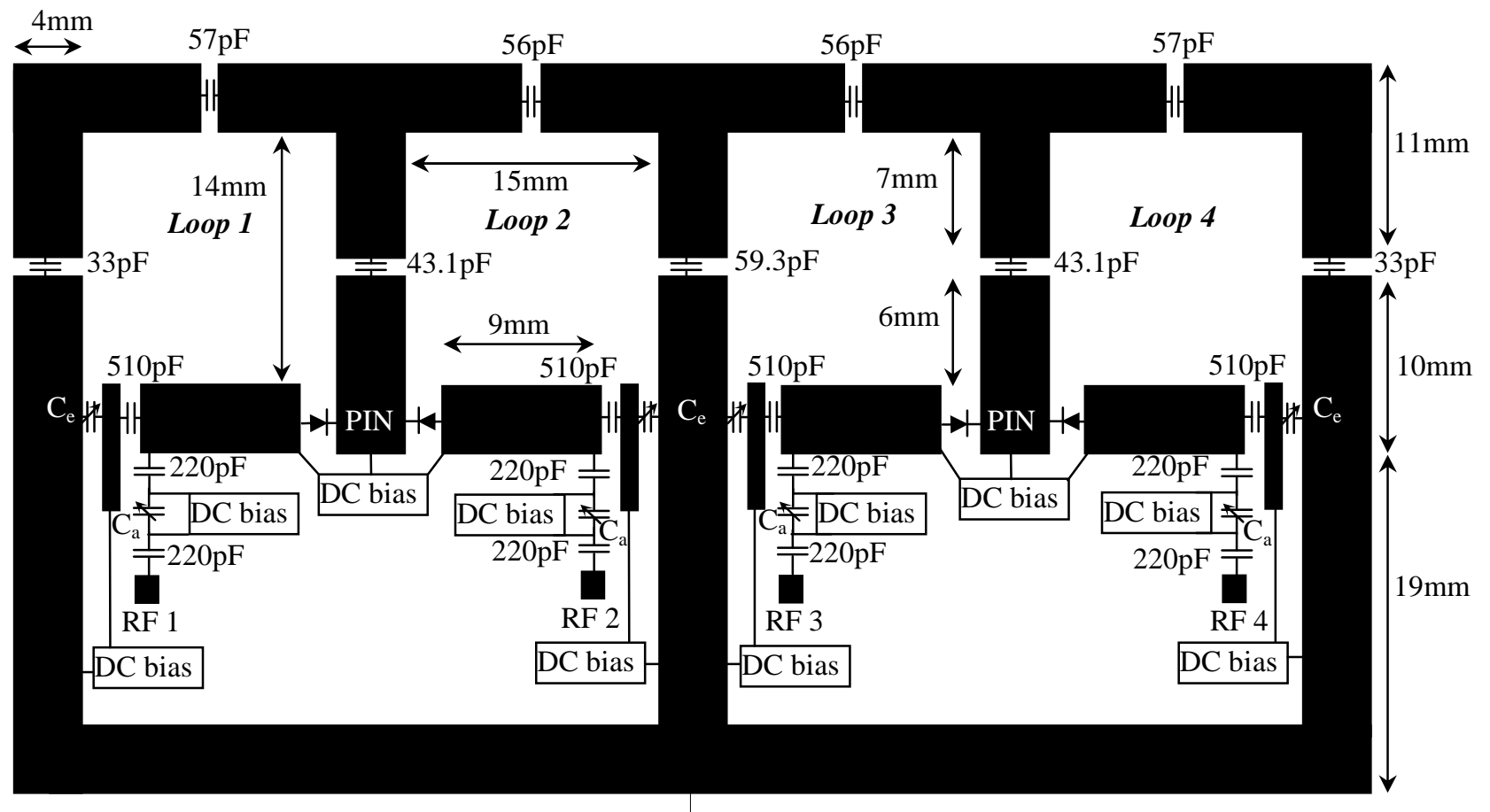

Fig. 4. RF layout of the four-channel coil array with lumped components values. 
loop to ensure tuning, matching and actively decoupling. This bias box was designed to be interfaced with the "coil interface unit (CIU)" from the Bruker system (Advance I and II) or with an additional external power source. CIU is used to drive DC voltages during MRI experiment while the external power source is used to drive DC voltages during the coil array building and characterization with a Vector Network Analyzer (VNA) in the electronic room lab.

\section{CHARACTERIZATION}

\section{A. Circuit Simulations}

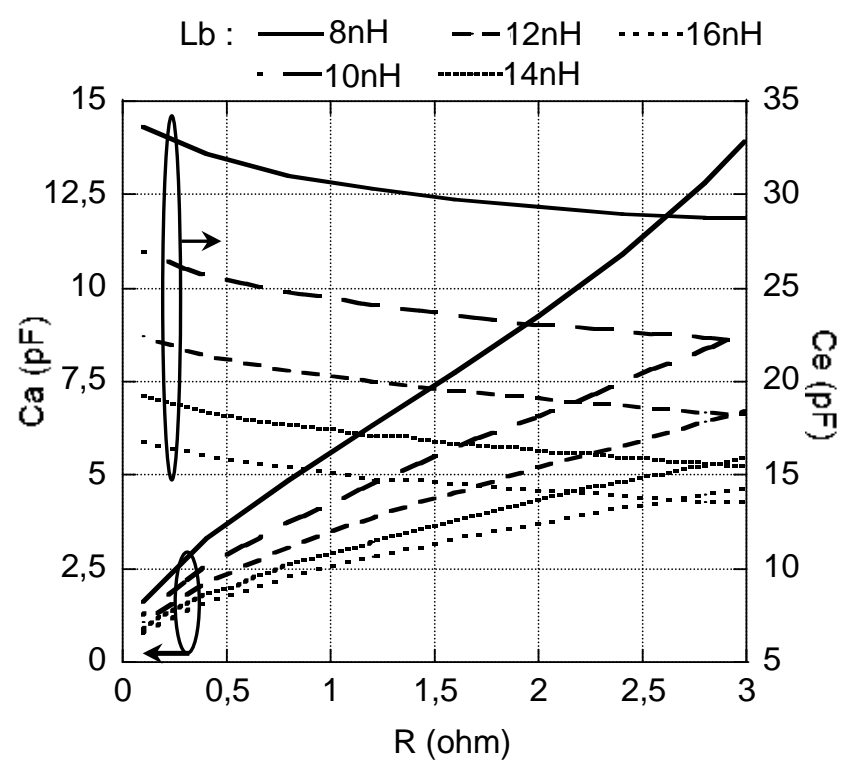

Fig. $5 C_{a}$ and $C_{e}$ capacitance values versus $R$ and $L_{b}$ values.

In this section, circuit simulations of the four-channel coil array topology (Fig. 2) are presented so as to confirm the equivalent electrical circuit. These simulations were achieved for arbitrarily chosen values of coil components: the $L_{b}, L_{c}$ and $L_{d}$ inductors were fixed to the same $10 \mathrm{nH}$ value to constitute four perfect square loops. The $C_{c}$ and $C_{d}$ capacitance values were calculated from equation (1) at an operating frequency $f_{0}=300 \mathrm{MHz}: C_{c}=C_{d}=28.14 \mathrm{pF}$ while $C_{a}$ and $C_{e}$ values, calculated from the equations (2) and (3), ensured matching of the loops to $50 \Omega$ at $f_{0}=300 \mathrm{MHz}$. $C_{a}$ and $C_{e}$ capacitance values versus $R$ and $L_{b}$ values are shown in Fig. 5. For circuit simulations the $R$ resistor is fixed to $1.1 \Omega$ which is a coherent practical value using similar components (extracted from previous works [20]). This $R$-value fixed $C_{a}$ to $4.5 \mathrm{pF}$ and $C_{e}$ to $24.2 \mathrm{pF}$. The circuit of Fig. 2 was simulated with Designer software (Ansys, USA). Results of S-parameters are shown in Fig. 6; passive circuit symmetry implies that some Sparameters are similar. For more clarity, these similar parameters are represented by the same symbol on simulation results (see Fig. 6 legend). In practice, when a two-channel coil using varicap diodes and its bias circuits is realized, the parasitic inductance $L_{\text {gnd }}$ connected between the ground plane and the loops deteriorates the S-parameter values [20]. Fig. 7 presents S-parameter results of the four-channel coil array by adding three parasitic inductors $L_{\text {gnd }}$ between the ground plane and inductors $L_{c}$. The values of these parasitic inductors $L_{g n d}$ were fixed to $5 \mathrm{nH}$ in Fig. 7 a) and to $10 \mathrm{nH}$ in Fig. 7 b); All other component values remain identical to simulations that results are shown in Fig. 6.

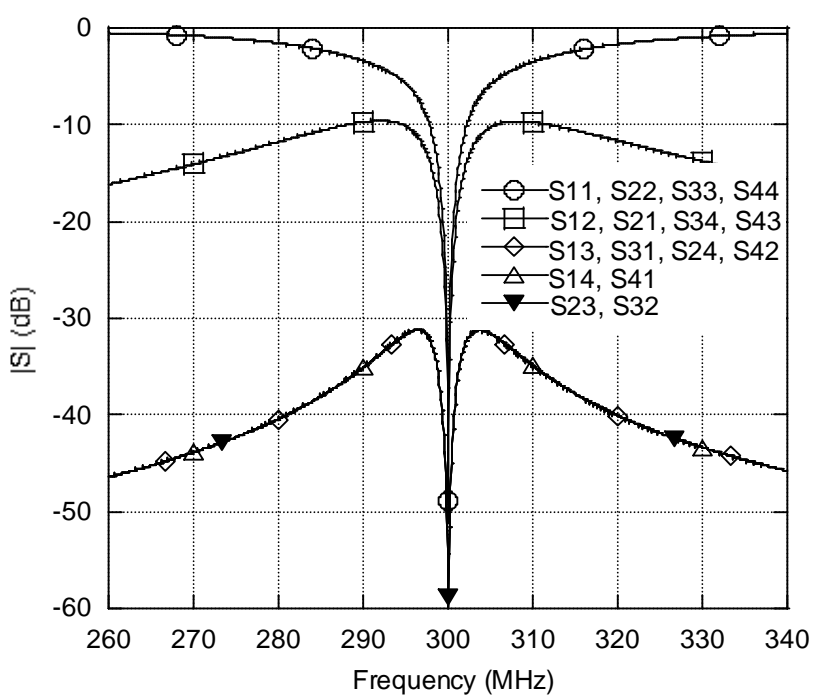

Fig. 6 Simulations of the four-channel coil array.

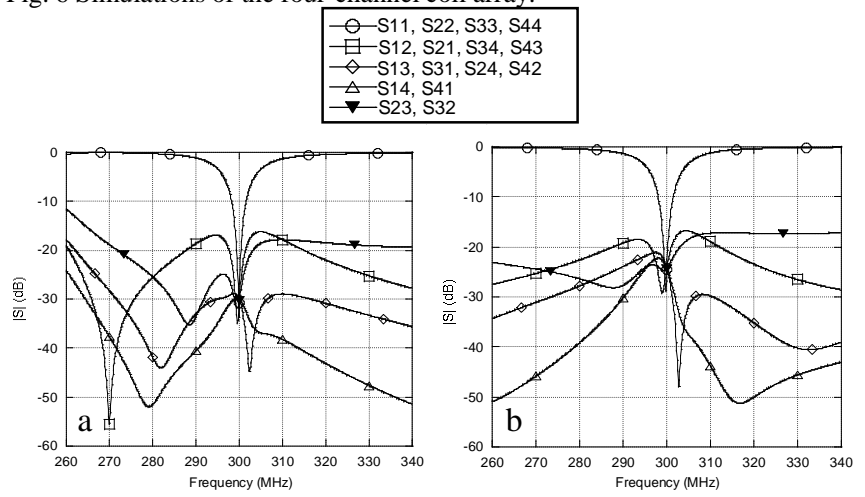

Fig. 7 Simulations of the four-channel coil array with parasitic inductances equal to a) $5 \mathrm{nH}$, b) $10 \mathrm{nH}$.

Fig. 6 presents simulations of the ideal circuit: at $f_{0}=300 \mathrm{MHz}\left|S_{i i}\right|$ parameters smaller than $-20 \mathrm{~dB}$ illustrate the good matching of each loop to $50 \Omega$ while $\left|S_{i j}\right|$ parameters smaller than $-20 \mathrm{~dB}$ demonstrate the good decoupling between elements. Quality factor extracted from simulations is 26 for all loop of coil array. In Fig. 7, the presence of parasitic inductors modified the $|S|$ parameters on a wideband around the operating frequency; quality factors extracted from simulations are 55 in Fig. 7 a) and 54 in Fig. 7 b) for all loop of coil array. At the operating frequency, all $\mathrm{S}$ parameters were inferior to $-50 \mathrm{~dB}$ without $L_{\text {gnd }}$, inferior to $-30 \mathrm{~dB}$ with $L_{\text {gnd }}=5 \mathrm{nH}$ and inferior to $-24 \mathrm{~dB}$ with $L_{\text {gnd }}=10 \mathrm{nH}$. Comparing S-parameters in figures 6 and 7 bring the following observations:

- Loop quality factor is better in presence of the parasitic inductors $L_{\text {gnd }}$. Indeed quality factor values are 55 and 54 when $L_{\text {gnd }}$ is equal to $5 \mathrm{nH}$ and $10 \mathrm{nH}$, respectively. Without $L_{\text {gnd }}$-value, the quality factor is 26 .

- Decoupling parameters between loop 1 and 2 (or loop 3 and 4) are improved over all frequencies, except the operating frequency, in presence of $L_{\text {gnd }}$ with $\left|S_{12}\right|<-9$ dB (Fig. 6) to $\left|S_{12}\right|<-16 \mathrm{~dB}$ (Fig. 7). 
- Transmission parameters minima no longer occur at the same frequency.

- All minimum transmission parameters are deteriorated in presence of $L_{\text {gnd }}$ due to the perfect ground transformation into inductive impedance.

In spite of the S-parameters deterioration at the operating frequency, the four-channel coil array keeps good matching for each loop and acceptable transmission parameters (with $\left|S_{i j}\right|<-20 \mathrm{~dB}$ ) when parasitic inductors are inferior to $14 \mathrm{nH}$.

\section{B. $\quad|S|$ Parameters Measurements}

Measurements of the four-channel surface coil array design in section III were carried out with an Agilent E5071C fourport VNA (Agilent Technologies Inc., Santa Clara, CA, USA). The coil array was matched in loading conditions using two cylindrical phantoms filled with saline water solution $\left(\mathrm{NiSO}_{4}\right.$ $1.25 \mathrm{~g} / \mathrm{L}$ and $\mathrm{NaCl} 5 \mathrm{~g} / \mathrm{L}$ ) to mimic rat legs. Each loop was independently matched to $50 \Omega$ at $f_{0}=300 \mathrm{MHz}$ using the bias box.

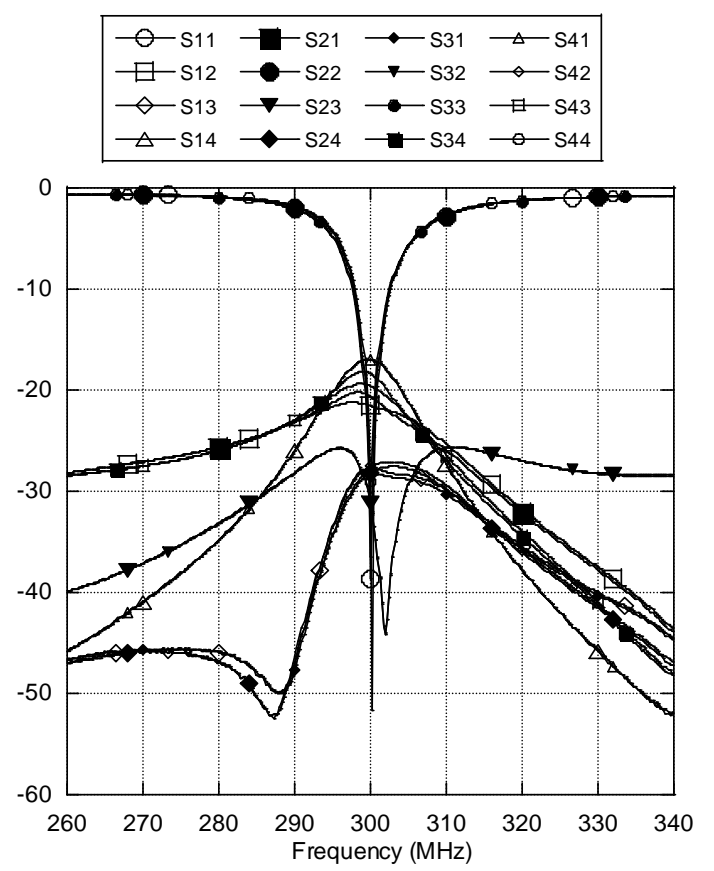

Fig. $8|\mathrm{~S}|$ parameters measurements of the four-channel NMR coil array.

Fig. 8 shows the sixteen $|S|$ parameter measurements realized when PIN diodes are activated. Each curve symbol represents a group of curves which are similar for a perfect device (as in Fig. 6). In practice, the circuit is not perfectly symmetric: curves are different inside a group. These curve differences are represented by different size and grey level of the same symbol. Bias voltages applied to each varicap diode and their capacitance values extracted from datasheet are summarized in Table I for loaded and non-loaded coil. We note a significant bias voltage difference between internal ( 2 and 3 ) and external (1 and 4) loops but non significant difference between loaded and non-loaded coil. Values of tuning and matching diodes capacitance allow extraction of $L_{b}$ and $R$ values from Fig. 5 using $C_{a}$ and $C_{e}$ curves. $C_{a}=6 \mathrm{pF}$ and $C_{e}=24 \mathrm{pF}$ for external loops give $L_{b}$ and $R$ of about $9 \mathrm{nH}$ and $1.25 \Omega$, respectively.
$C_{a}=4 \mathrm{pF}$ and $C_{e}=16 \mathrm{pF}$ for internal loops give $L_{b} \approx 13 \mathrm{nH}$ and $R \approx 1.7 \Omega . L_{b}$ and $R$ differences between internal and external loops can be explained by three dimensional topology difference between the loops and the presence of different neighbor loops. These differences between internal and external loops illustrated the component adjustment difficulties for the coil realization. Difference of capacitance values between loaded and non-loaded coil is not significant to extracted magnetic loses. Circuit simulations, whereby differences in electromagnetic fields between loops are not taken into account, achieve similar results for all loops. $L_{b}$ and $R$ values extracted from Fig. 5 are coherent with design and TABLE I

TUNING AND MATCHING VOLTAGES OF EACH LOOP CAPACITANCE VALUE OF EACH VARICAP DIODE QUALITY FACTOR OF EACH LOOP

\begin{tabular}{|l|l|c|c|c|c|}
\multicolumn{7}{c|}{ QUALITY FACTOR OF EACH LOOP } \\
\hline Non-loaded & \multicolumn{1}{|c|}{ Loop } & 1 & 2 & 3 & 4 \\
& Tuning (V) & -0.23 & -1.29 & -1.17 & -0.26 \\
& Ce (pF) & 25 & 17 & 17 & 25 \\
\cline { 2 - 6 } & Matching (V) & -8.58 & -12.5 & -12.5 & -8.8 \\
& Ca (pF) & 6 & 4 & 4 & 6 \\
\cline { 2 - 6 } & Q & 43 & 45 & 43 & 44 \\
\hline Loaded & Tuning (V) & -0.26 & -1.34 & -1.22 & -0.3 \\
& Ce (pF) & 25 & 16 & 17 & 24 \\
\cline { 2 - 6 } & Matching (V) & -9.03 & -12.4 & -12.2 & -8.39 \\
& Ca (pF) & 6 & 4 & 4 & 6 \\
\cline { 2 - 7 } & Q & 42 & 40 & 41 & 43 \\
\hline
\end{tabular}

components of the coil.

Measurements results of Fig. 8 are in good agreement with simulated results when the parasitic inductors are added in the electrical circuit (Fig. 7): transmission parameters are inferior over all frequencies; $\left|S_{i j}\right|$ minima values are not superimposed at the same frequency and loops quality factors are improved compared to Fig. 6 without parasitic inductors. Measured $\left|S_{i i}\right|$ parameters show a coil matching for each loop better than -30 $\mathrm{dB}$ at $300 \mathrm{MHz} ; \quad\left|S_{i j}\right|$ parameters better than $-17 \mathrm{~dB}$ characterizing a good enough decoupling between loops pairs. Measured quality factors of each loop were reported on Table I for loaded and non-loaded coil array: they vary between 40 and 45. Quality factors were measured for the four channels with $\left|S_{i i}\right|=-3.3 \mathrm{~dB}$. This $-3.3 \mathrm{~dB}$ value correct for the $-0.3 \mathrm{~dB}$ losses present on $\left|S_{i i}\right|$ parameters over all frequencies; these losses provide by coaxial cables. The measured quality factors are low due to the small inductor $L_{b}$ and due to the series resistance of all lumped elements (six fixed capacitors, two varicaps and one PIN diode for each loop). Indeed, these quality factors, varying between 40 and 45 , have the same order of magnitude that simulated results using a $1.1 \Omega$ estimated resistance $R, 10 \mathrm{nH}$ loop inductors $L_{b}, L_{c}, L_{d}$ and with a parasitic inductors $L_{g n d}$ inferior to $5 \mathrm{nH}(Q=55)$.

Even though components in the experimental setup do not exactly match values used in simulations and even though electromagnetic field is not taken into account in circuit simulations, experimental result curves are similar to predicted simulated results. The presence of parasitic inductances is confirmed by the experimental measurements. The elementary equivalent electrical circuit in Fig. 2 is sufficient to explain fundamental operations of the coil array but more complex circuit of electromagnetic simulations will be necessary if perfect adjustment between simulated and measured data is required. 


\section{Magnetic Resonance Imaging}

The experiments were performed on a Bruker 7T Biospec MR scanner (Bruker, Ettlingen, Germany). The maximum gradient amplitude available is $400 \mathrm{mT} / \mathrm{m}$ and the clear bore diameter is of $120 \mathrm{~mm}$. In vitro acquisitions were performed to compare two coils: the designed four-channel coil array and a transmit/receive quadrature volume coil (32 mm, RAPID Biomedical GmBH, Rimpar, Germany). A transmit birdcage coil with $112 \mathrm{~mm}$ outer diameter and $72 \mathrm{~mm}$ inner diameter (Rapid Biomedical, Würzburg, Germany) is used for transmission when the designed four-channel coil array is used as receiver. In vivo acquisitions were performed simultaneously on both knee joints of a rat.

1)

$$
\text { In vitro }
$$
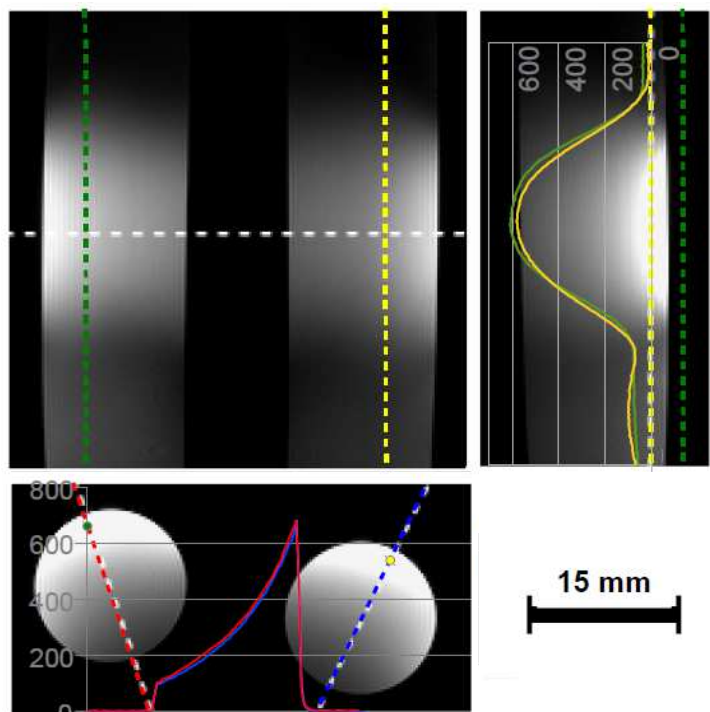

Fig. 9 Coronal, sagittal and axial images obtained on the phantoms with SNR profiles.

In order to characterize the designed coil array (signal distribution and SNR), experimental tests were performed on $15 \mathrm{~mm}$ inner diameter cylindrical phantoms filled with salty water $\left(\mathrm{NiSO}_{4} 1.25 \mathrm{~g} / \mathrm{L}\right.$ and $\left.\mathrm{NaCl} 5 \mathrm{~g} / \mathrm{L}\right)$ mimicking load conditions found on animal tissues. A 3D FLASH sequence was used with the following parameters: $15 \mathrm{~ms}$ TR, $6 \mathrm{~ms}$ TE and $50 \mathrm{kHz}$ receiver bandwidth. A total of 128 partitions (32.5 $\mathrm{mm}$ thick) were acquired with a FOV of $6.5 \times 6.5 \mathrm{~cm}^{2}$ and an acquisition matrix size of $256 \times 256$ in $8 \min 12 \mathrm{sec}(\mathrm{NA}=1)$.

Fig. 9 shows axial and coronal MR images obtained on the two uniform cylindrical phantoms. No visible susceptibility artifacts from the components were depicted. Fig. 9 shows also the SNR sagittal and coronal profiles obtained with the four-channel coil and along axes defined in the figure. These profiles can be compared in the figure with the homogenous SNR of about 200 obtained in the phantom with the quadrature volume coil. The maximum SNR gain for the fourchannel coil array was up to 3.4 times higher compared to the SNR obtained with the quadrature volume coil. Axial image of single channels presented in Fig. 10 shows the independence of the channels.
SNR profiles show an undesirable signal outside of the coil array. This signal is due to the loop of the ground plane (Fig. 4). Amelioration of this point would be a further stage to improve the coil array.
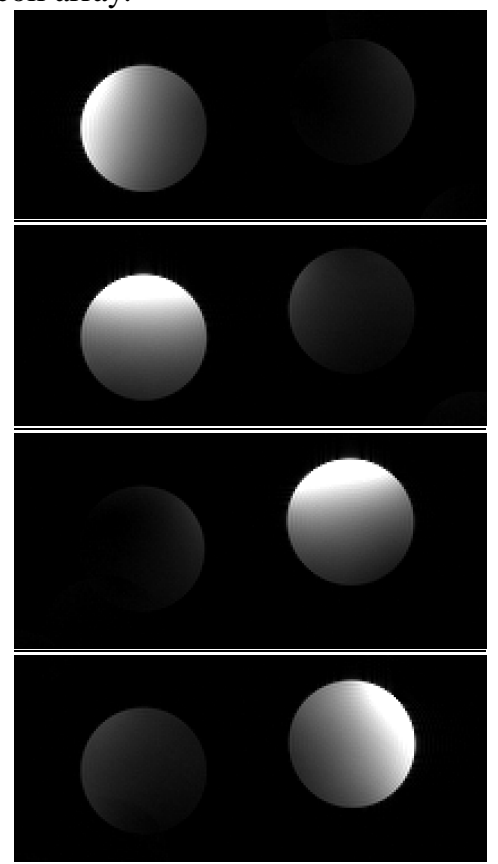

Fig. 10 Axial images of each channel.

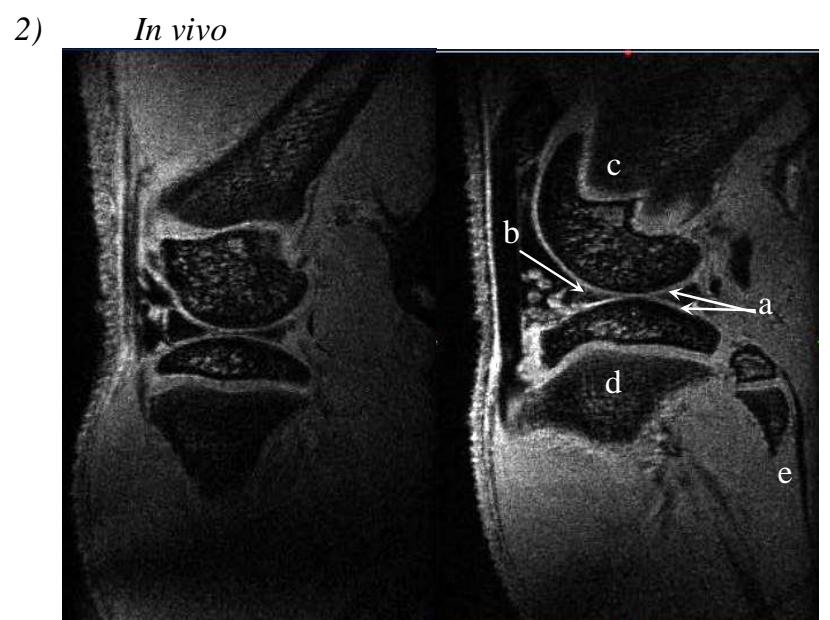

Fig. 11 Rat knee MR imaging. a) Articular cartilage, b) Meniscus, c) Distal femur, d) Proximal tibia, e) Fibula.

A quick gradient-echo localizer with three orthogonal plane orientations of $6 \times 6 \mathrm{~cm}^{2}$ FOV was first run to identify the region of interest and to allow graphical prescriptions of the subsequent scans. Rapid Acquisition with Relaxation Enhanced (RARE) sequences in sagittal, axial and coronal plans were performed in order to check that the knee joints were placed adequately with respect to the four-channels receiver coil array and to carefully prescribe the following high-resolution (HR) sagittal 3D slabs orthogonally to the anteroposterior flexion axis of the knees [23]. The 3D HRMRI of the rat knees were obtained using a 3D FLASH sequence with the following parameters: $30^{\circ}$ flip angle, $50 \mathrm{~ms}$ 
TR, $3.6 \mathrm{~ms}$ TE, $27.8 \mathrm{kHz}$ receiver bandwidth. Anti-aliasing was used in both slice and phase encoding direction. A total of 256 partitions (98 $\mu \mathrm{m}$ thick) were acquired using a double slab volume selection, one for each knee, with a FOV of $1.25 \mathrm{x}$ $1.88 \mathrm{~cm}^{2}$ and an acquisition matrix size of $256 \times 384$. Due to the simultaneous signal acquisition on four channels, the amount of data exceeded 4GBytes. The proprietary software (Paravision 5.1, Bruker Biospin, Germany) being run on a 32bits operating system, online image reconstruction could not be performed. Thus, we employed a home-made script (Matlab 7.13a, Mathworks, Milwaukee, USA) to finally obtain the reconstructed slices (in-plane pixel: 49 x $49 \mu^{2}$, thickness: $98 \mu \mathrm{m}$ ). This script consists to open and arrange Bruker files of raw data in multidimensional matrices. Data were preprocessed before fast Fourier transformation. They were first reordered according to $\mathrm{k}$-space position, coil element and volume to generate a 5D volume $(512 \times 512 \times$ 256) voxels $\times 4$ coils $x 2$ slabs and including data oversampling factors $(2.0 ; 1.3 ; 2.0)$ used to avoid image folding due to small FOV. Hamming window and a 3D FFT were applied for each coil element and slab volumes. Then, the spatial volume was cut down to 256 x 384 x 128 pixels. Finally, each slab was reconstructed using a sum of square of the relevant coils (coil $1 \& 2$ for right knee, $3 \& 4$ for left knee). The acquired volumes of $1.25 \times 1.88 \times 1.25 \mathrm{~cm}^{3}$ completely covered each rat knee. The scan time for the FLASH sequence was $1 \mathrm{~h} 22 \mathrm{~min}$.

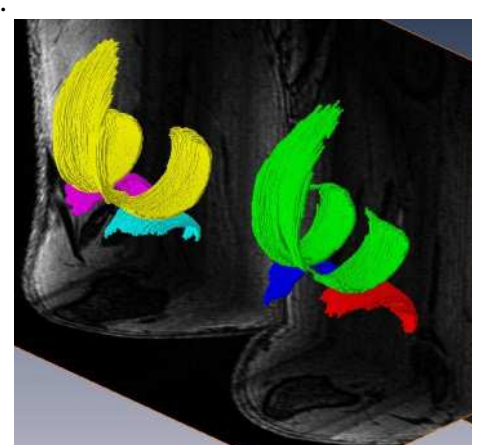

Fig. 12 Segmented cartilage compartments of both rat knees performed on HR-MR images. Femoral cartilages appear in yellow and green colors, lateral tibial cartilages apnear in nink and red colors, medial tibial cartilage appear in tourquois and blue colors.

Fig. 11 presents the image reconstruction of the right and left knee, respectively. The 3D-MRI data sets were used to compute the femoral condylar groove as well as medial and lateral tibial plateaus cartilage volumes. Segmented cartilage compartments of both rat knees are presented in Fig. 12. The knee cartilage compartments were drawn directly on interactive touch-sensitive screen this screen using the pen provided. Manual contouring and pixel counting were done using AMIRA software.

To our knowledge, the voxel size reported here represents the highest spatial resolution performed in vivo on both knee joints of a rat for an acquisition time of less or equal to $1 \mathrm{~h} 22 \mathrm{~min}$. The SNR gain obtained with the multi-channel coil was used to improve the spatial resolution [24].

\section{CONCLUSION}

An equivalent electrical circuit of a four-channel NMR surface coil array based on common conductor decoupling technique was proposed. The topology allowed good matching and good decoupling between elements without the use of additional low input impedance preamplifier.

The design of a four-channel surface coil array with a particular wave-like topology for the simultaneous two rat knees imaging was realized at $300 \mathrm{MHz}$. The capacitor adjustments permit to obtain decoupling better than $-17 \mathrm{~dB}$ between all elements pairs. To our knowledge, this coil array represents the first four-channel NMR surface coil array based on common conductor not using the preamplifier decoupling technique. Performance of the coil array was proved through the simultaneous HR-MRI of both knees joint of a rat. Voxel size of $49 \times 49 \times 98 \mu^{3}$ was obtained for a $1 \mathrm{~h} 22 \mathrm{~min}$ acquisition time. In future works, similar four-channel coils array build with mechanical variable capacitors could be used as transceiver coil. In this case each channel phase and magnitude could be studied and electromagnetic simulations using finite element method or method of moments performed so as to look for the magnetic field homogeneity.

\section{REFERENCES}

[1] P. B. Roemer, W. A. Edelstein, C. E. Hayes, S. P. Souza and O. M. Mueller, "The NMR phased array," Magn Reson Med, vol. 16, pp. 192225, 1990.

[2] D. Gareis, T. Wichmann, T. Lanz, G. Melkus, M. Horn, and P. M. Jakob, "Mouse MRI using phased-array coils," NMR Biomed, vol. 20, pp. 326-34, 2007

[3] J. B. Kneeland and J. S. Hyde, "High-resolution MR imaging with local coils," Radiology, vol. 171, pp. 1-7, 1989.

[4] K. P. Pruessmann, M. Weiger, M. B. Scheidegger, and P. Boesiger, "SENSE: sensitivity encoding for fast MRI," Magn Reson Med, vol. 42, pp. 952-62, 1999.

[5] M. A. Griswold, P. M. Jakob, R. M. Heidemann, M. Nittka, V. Jellus, J. Wang, B. Kiefer, and A. Haase, "Generalized autocalibrating partially parallel acquisitions (GRAPPA)," Magn Reson Med, vol. 47, pp. 120210, 2002.

[6] P. Angelidis, K. Vassiliadis, and G. D. Sergiadis "Lowest Mutual Coupling Between Closely Spaced Loop Antennas," IEEE Trans Antennas and Propag, vol. 39, pp. 949-953, 1991.

[7] W. E. Kwok, J. Zhong, Z. You, G. Seo and S. M. S. Totterman, "A fourelement phased array coil for high resolution and parallel MR imaging of the knee," Magn Reson Imag, vol. 21, pp. 961-967, 2003.

[8] N. De Zanche, J. A. Massner, C. Leussler and K. P. Pruessmann, "Modular design of receiver coil arrays," NMR Biomed, vol. 21, pp. 644$654,2008$.

[9] A. Reykowski, S. M. Wright and J. R. Porter, "Design of Matching Networks for Low Noise Preamplifiers," Magn Reson Med, vol. 33, pp. 848-852, 1995.

[10] G. Adriany, P. F. Van de Moortele, J. Ritter, S. Moeller, E. J. Auerbach, C. Akgun, C. J. Snyder, T. Vaughan and K. Ugurbil, "A geometrically adjustable 16-channel transmit/receive transmission line array for improved RF efficiency and parallel imaging performance at 7 tesla," Magn Reson Med, vol. 59, pp. 590-597, 2008.

[11] R. G. Pinkerton, E. A. Barberi and R. S. Menon, "Noise properties of a NMR transceiver coil array," Journal of Magnetic Resonance, vol. 171, pp. 151-156, 2004.

[12] B. K. Li, F. Liu and S. Crozier, "Focused, eight-element transceiver phased array coil for parallel magnetic resonance imaging of the chestTheoretical considerations," Magn Reson Med, vol. 53, pp. 1251-1257, 2005.

[13] R. G. Pinkerton, E. A. Barberi and R. S. Menon, "Tranceiver surface coil array for magnetic resonance imaging of the human brain at $4 \mathrm{~T}$," Magn Reson Med, vol. 54, pp. 499-503, 2005.

[14] X. Zhang and A. Webb, "Design of a capacitively decoupled transmit/receive NMR phased array for high field microscopy at 14.1 T," Journal of Magnetic Resonance, vol. 170, pp. 149-155, 2004.

[15] J. Wang, "A novel method to reduce the signal coupling of surface coils for MRI”. In: Proc 14th Annual Meeting ISMRM, Seattle; 1996. 
[16] D. Gareis, T. Neuberger, V. C. Behr, P. M. Jakob, C. Faber, M. A. Griswold "Transmit-receive coil-arrays at 17.6T, configurations for H-1, Na-23, and P-31 MRI," Concepts Magn Reson Part B, Magn Reson Eng, vol. 29B, pp. 20-27, 2006

[17] M. A. Dieringer, W. Renz, T. Lindel, F. Seifert, T. Frauenrath, F. von Knobelsdorff-Brenkenhoff, H. Waiczies, W. Hoffmann, J. Rieger, H. Pfeiffer, B. Ittermann, J. Schulz-Menger, and T. Niendorf, "Design and Application of a Four-Channel Transmit/Receive Surface Coil for Functional Cardiac Imaging at 7T" Journal of Magnetic Resonance Imaging, vol. 33, pp. 736-741, 2011.

[18] Lanz, T. Steinberger, S. Dodd, F. F. Paiva, A. C. Silva, A. P. Koretsky, “ 4 channel rat head arrays for $7 \mathrm{~T}$ and $11.7 \mathrm{~T}$," ISRMRM, pp. 2589, 2006.

[19] A. Rengle, M. Armenean, R. Bolbos, J. C. Goebel, A. Pinzano-Watrin, H. Saint-Jalmes, P. Gillet and O. Beuf, "A dedicated two-channel phased array receiver coil for high resolution MRI of the rat knee cartilage at 7T,” IEEE Trans Biomed Eng, vol. 56, pp. 2891-2897, 2009.

[20] A. L. Perrier, D. Grenier, A. Pouzin, F. Esclassan, N. Ravel, P. Litaudon, O. Beuf, "Design of a two-channel NMR coil using an impedance transformation approach," IEEE Sensors Journal, vol. 12, Issue 6, pp. 1801-1808, 2012.

[21] F. D. Doty, G. Entzminger, J. Kulkarni, K. Pamarthy, and J. P. Staab "RF Coil Technology for Small-Animal MRI," NMR Biomed, vol. 20, pp. 304-325, 2007.

[22] D. I. Hoult "The NMR receiver: a description and analysis of design," Progess in NMR Spectroscopy, vol. 12, pp. 41-77, 1978

[23] C. Glaser, R. Burgkart, A. Kutschera, KH. Englmeier, M. Reiser and F. Eckstein, "Femorotibial cartilage metrics from coronal MR image data: Technique, test-retest reproducibility, and findings in osteoarthritis," Magn Reson Med, vol. 50, pp. 1229-1236, 2003.

[24] R. Bolbos, H. Benoit-Cattin, J.B Langlois, A. Chomel, E. Chereul, C. Odet, M. Janier, M. Pastoureau and O. Beuf, "Measurement of knee cartilage thickness using MRI: a reproducibility study in a meniscectomized guinea pig model of osteoarthritis, " NMR Biomed, vol. 21 , pp. 366-375, 2008.

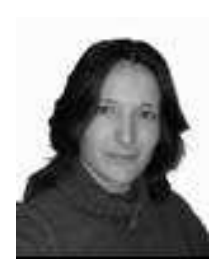

Anne-Laure Perrier was born in France in 1980. She received the M.Sc. degree in Optics, Optoelectronics, and Microwaves from the INPG ("Institut National Polytechnique de Grenoble"), Grenoble, France, in 2003. She received the Ph.D. degree in 2006 from the Laboratory of Microwaves and Characterization (LAHC), University of Savoie, France. Her research interests include the theory, design, and realization of tunable-impedance transformers and tunable power

dividers.

She has been an Assistant Professor since September 2008 at Claude Bernard University (Lyon, France), where she teaches electronics and signal processing. She continues her research at the Research Center on Medical Imaging (CREATIS). She designs and realizes RF sensors for MRI (Magnetic Resonance Imaging) applications.

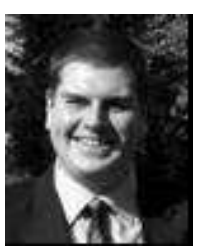

Jean-Christophe Goebel was born in Metz, France, in 1980. He received the M.Sc. degree in Bioengineering and Biomaterials from the Henri Poincaré University (Nancy, France) in 2004. Thereafter, he received the $\mathrm{Ph} . \mathrm{D}$. degree in Cell and Tissue Bioengineering from the Nancy Université (France) in 2009. Since 2010, he is working on a post-doctoral position at the Research Center on Medical Imaging (CREATIS UMR CNRS 5220, Lyon, France). His actual research interests deal with the MRI morphological and structural characterization of implantable chondrogenic biomaterials.

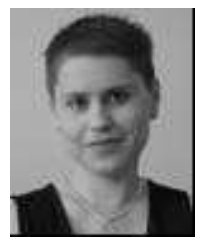

Astrid Pinzano-Watrin was born in France in 1975. She received her $\mathrm{PhD}$ degree in drug science in 2001 from Henri Poincaré University (Nancy, France) concerning the influence of matrix components on MRI cartilage signal. She performed postdoctoral position at the University of Bern working on the cartilage tissue engineering and the influence of mechanical loading. She joined the 7561 CNRS research unit in 2003 as CNRS researcher. Her research works concerned the cartilage tissue engineering with stem cells and the MRI morphological and structural study of articular cartilage.

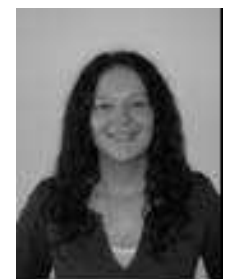

Emilie ROEDER was born in France in 1986. She received her MSc degree in Bioengineering from the Henri Poincaré University (Nancy, France) in 2010. Since 2009, she has been working at the UMR 7561 CNRS research unit (Laboratoire de Physiopathologie et Ingénierie Articulaires, Nancy) within the framework of her $\mathrm{PhD}$ in Bioengineering. Her research works concerned the cartilage tissue engineering with stem cells and the MRI morphological and structural study of articular cartilage.

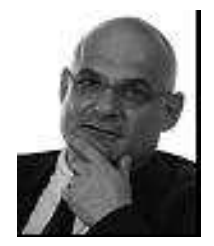

Pierre GILLET was born in Alger, Algeria, in 1960. He received his $\mathrm{MD}$ in 1988 and his $\mathrm{PhD}$ in 1996. He is actually Rheumatologist and Professor of Pharmacology in the School of Medicine, in Nancy I University. He is in charge of the group "Bioingénierie Tissulaire, Vectorisation \& Imagerie Articulaire" in the Unit of Reseearch "Physiopathologie \& Pharmacologie Articulaires", UMR 7561 CNRS-Nancy I, directed by Jacques MAGDALOU. He is mostly involved in tissular characterization of the cartilage, experimental models of $\mathrm{OA}$ and tissular engineering of cartilage.

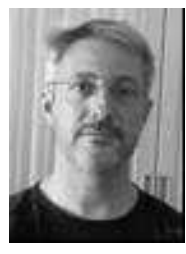

Denis Grenier is born in France in 1968. After a master in physics he received a $\mathrm{PhD}$ in biomedical engineering from Université Claude Bernard Lyon 1999. Post doctoral fellow at University of California San Francisco, he worked between 1999 and 2001 on MR sequence programming in the field of musculoskeletal application for UCSF radiology department and the field of Multiple Sclerosis for the neurology department. Since 2001 he is in charge of CREATIS UMR CNRS 5012 MR facility. He is part of the team "NMR and optics: Methods and systems". His research focuses on MR signal creation.

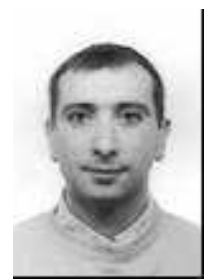

Olivier Beuf was born in France in 1970. He received his $\mathrm{PhD}$ degree in physics from the University de Lyon in 1998. From 1998 to 2000 he was a postdoctoral fellow at the University of California, San Francisco working on advanced methodological MRI developments for musculoskeletal applications. After a short position as MRI application specialist at Siemens Medical Solutions, France, he joined the CNRS 5012 NMR laboratory in 2000. Today, he is the head the research team entitled "NMR and optics: Methods and systems" from the CREATIS laboratory. His research mainly focuses on radiofrequency coils and sequence developments for medical or biological MRI applications both on human and small animals. 